\section{ÜBER DEN EINFLUSS PATHOLOGISCHER ZUSTÄNDE AUF DIE ZERSTÖRUNG DES ADRENALINS'1).}

\author{
Von
}

\section{A. BÁlint und L. Goldschmidt.}

Wie verschieden auch das Adrenalin bei den verschiedenen Individuen wirkt, allen Adrenalinwirkungen gemeinsam ist ihre Flüchtigkeit. Das Adrenalin wird aus dem Organismus nicht ausgeschieden. Wir müssen folgern, daß es im Körper zerstört oder umgebaut wird. Es wurde schon vielfach angenommen, daß die flüchtige Wirkung des Adrenalins durch die rasche Zerstörung bedingt ist. Bei der Zerstörung des Adrenalins kommen zwei Faktoren besonders in Betracht. Erstens das Alkali, zweitens die Oxydation. Diese zwei Faktoren ändern sich bei dem Menschen unter gewissen pathologischen Zuständen und unsere Versuche richteten sich dahin, ob es einen bestimmten Zusammenhang zwischen der Wirkung und Zerstörung des Adrenalins und gewissen pathologischen $\mathrm{Zu}$ ständen gibt. Dabei kommen Zustände in Betracht, bei denen die Stoffwechselprozesse und der Chemismus der Gewebe von der Norm abweichen.

Um den Einfluß des Alkaligehaltes des Körpers auf den Ablauf der Adrenalinwirkung zu studieren, sind die Säuglinge besonders geeignet. Thre Alkalireserve ändert sich schnell, so daß sie leicht in acidotischen Zustand kommen. Die Blutdrucklkurve, als $\mathrm{Ma} \beta$ der Adrenalinwirkung wird ferner bei dem Säugling nicht von so vielen Komponenten beeinflußt, wie beim Erwachsenen oder größeren Kinde. Wir haben besonders die Rücklaufzeit, d. h. die Zeit berücksichtigt, in der der Blutdruck wieder seine normalen Werte erreicht.

Bei klinischen Zeichen der Acidose, wo wir im Liquor cerebrospinalis oder im Blute einen niedrigen $\mathrm{NaHCO}_{3}$-Grehalt fanden, haben wir Adrenalin-Blutdruckkurven aufgenommen. Die Rücklaufzeit der Blutdruckkurven war immer deutlich verlängert. Sie betrug $4 \mathrm{O}-5 \mathrm{O}$ Minuten gegenüber $20-25 \mathrm{Minu}-$ ten der Norm. In manchen Fällen vermuteter Acidose war die Rücklaufzeit normal.

Die Frühgeburten zeichnen sich durch einen niedrigen Alkali-Gehalt des Blutes aus. Wir fanden, daß die Rücklaufzeit der Blutdruckkurve bei Frühgeburten deutlich verlängert ist.

Es.lag nun nahe, Fälle zu untersuchen, bei denen eine Alkalose anzunehmen ist. Wir untersuchten fiebernde Säuglinge, weil in der letzten Zeit behauptet wurde, daß bei infektiösen, fieberhaften Prozessen eine Alkalose besteht. Wir haben nun diese angebliche Alkalose in den meisten unserer untersuchten Fieberfällen vermißt. (Messung des $\mathrm{NaHCO}_{3^{-}}$ Gehaltes durch die Rohonyi sche Methode.) Die Rücklaufzeit der Adrenalinblutdruckkurve zeigte dagegen eine sehr interessante Gesetzmäßigkeit; sie war nämlich stets stark verkürzt. Sie betrug immer nur $4-8$ Minuten, und die gesamte Wirkung des Adrenalins war auch meistens herabgesetzt. Diese Gesetzmäßigkeit läßt sich auch beim größeren Kinde nachweisen. Wir haben dieselben Kinder im fieberhaften und fieberfreien Zustande untersucht. Kurve I ist die Blutdruckkurve eines normalen Säuglings, Kurve II die Blutdruckkurve eines acidotischen und Kurve III eines fiebernden Säuglings.

Im veränderten Alkaligehalt des Blutes haben wir also keine genügende Erklärung für die verlängerte bzw. verkürzte Rücklaufzeit gefunden. Dies war auch vorauszusehen, da die Wasserstoffzahl immer dieselbe bleibt. Wir müßter also den zweiten Faktor, die Oxydation in Betracht ziehen. Sie ist nämlich bei der Acidose abgestuft, beim Fieber gesteigert.

Um die Sachlage klinisch-experimentell näher zu klären, haben wir einen $\mathrm{Zustand}$ gewählt, wo die $\mathrm{CO}_{2}$-Spannung und der $\mathrm{NaHCO}_{3}$-Gehalt des Blutes bzw. des Organismus stark abgesunken ist. Dieser Zustand wäre die experimentelle Uberventilation. Hier besteht eine Alkalose, weil das $\mathrm{NaHCO}_{3}$ als Puffer nur bis zu einer gewissen Grenze abnehmen kann, dann ändert sich sogar die Wasserstoffzahl nach der alkalischen Seite. Bei der experimentellen Uberventilation wird

a) Erscheint ausführlich im Jabrbuch für Kinderheilkunde. sozusagen mehr $\mathrm{O}_{2}$ durch den Organismus geführt. Die Abnahme des $\mathrm{NaHCO}_{3}$-Gehaltes des Biutes konnten wir nachweisen. Kurve IV ist die Blutdruckkurve eines gröBeren Kindes, Kurve V die Blutdruckkurve desselben Kindes im Stadium der Uberventilation (I 5 Minuten tief atmen). Die Adrenalinwirkung ist also im Stadium der Utberventilation sehr deutlich herabgesetzt.

Die Adrenalinwirkung im menschlichen Organismus hängt von vielen $z$. $T$. unbekannten Faktoren $a b$. Wir sind nicht berechtigt auch von gesetzmäßigen Endresultaten auf einzelne Faktoren bindende Schlüsse zu ziehen. Um den Einfluß der oben erwähnten Prozesse (Alkali, Oxydation) rein darzustellen, haben wir die Zerstörung des Adrenalins in vitro und am Trendelenburgschen Froschpräparat studiert.

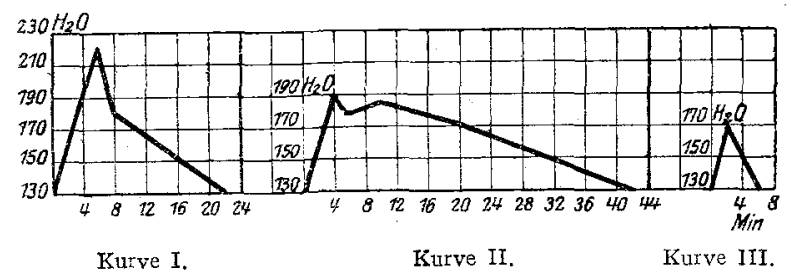

Unsere Stammlösungen waren Pufferlösungen, bestehend aus primärem und sekundärem Natriumphosphat, mit einer Wasserstoffzahl, die den Körperflüssigkeiten eigen ist. $\mathrm{Zu}$ diesen Lösungen wurde Adrenalin und $\mathrm{NaHCO}_{3}$ in der Menge von $\frac{2 n}{100}$ und $\frac{5 n}{100}$ zugesetzt. Dann haben wir die Pufferlösung mit physiologischer Kochsalzlösung, das $\mathrm{NaHCO}_{3}$ mit gleicher Äquivalentmenge von $\mathrm{Na}_{2}-\mathrm{CO}_{3}$ ersetzt. Wir haben immer die Wirkung von $0,000_{5} \mathrm{mg}$ Adrenalin geprüft. Um den Einfluß der Oxydation auf die Zerstörung des Adrenalins klarer zu sehen, haben wir $\mathrm{O}_{2}$ durch die Adrenalin-Pufferlösung mit und ohne Alkalizusatz durchgeleitet. Wir wollen unsere Resultate tabel-

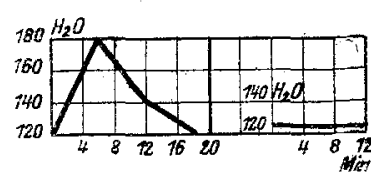

Kurve IV. Kurve $V$. larisch wiedergeben. Die volle Adrenalinwirkung bezeichnen wir mit: +++ , die abgeschwächte mit: ++ , die stark abgeschwächte mit: + und die fehlende mit: -.

\begin{tabular}{|c|c|c|c|c|}
\hline Löstung & $\begin{array}{c}\text { Nach } \\
1 / 2 \\
\text { Stunde }\end{array}$ & $\begin{array}{c}\text { Nach } \\
2 \\
\text { Stunden }\end{array}$ & $\begin{array}{c}\text { Nach } \\
3 \\
\text { Stunden }\end{array}$ & $\begin{array}{c}\text { Nach } \\
24 \\
\text { Stunden }\end{array}$ \\
\hline 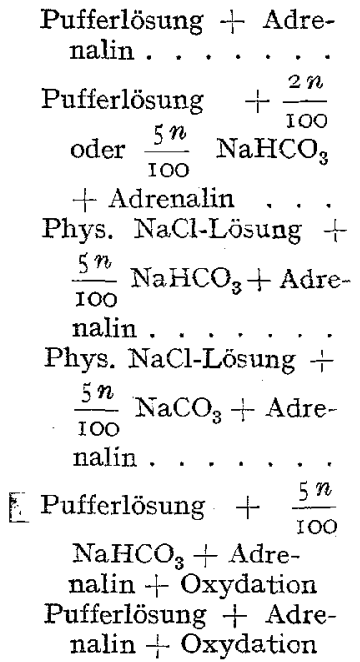 & $\begin{array}{c}1 \\
+1+1\end{array}$ & $+1+$ & $\frac{1}{1}++$ & $\frac{1}{1}+1$ \\
\hline
\end{tabular}

Die Puffer-Adrenalinlösung mit $\mathrm{Na}_{2}-\mathrm{HCO}_{3}$ wurde schon nach $1 / 2$ stündiger Oxydation deutlich rot.

1 Aus diesen Versuchen geht hervor, daß eine rasche Zerstörung des Adrenalins durch die Oxydation, und zwar nur 
in Gegenwart von Alkali vor sich geht. Auf Grund dieser Versuche ist es denkbar, daB im Fieber und bei der Überventilation die gesteigerte Oxydation die Ursache der kurzen Wirkung des Adrenalins ist. Bei den acidotischen Säuglingen dagegen können wir für die verlängerte Zerstörung und Wirkung des
Adrenalins außer den abgestuften oxydativen Prozessen auch die Verminderung der Blutalkalien in Betracht ziehen.

Als praktisches Ergebnis heben wir hervor, daß die Adrenalinwirkung im Fieber stark verkürzt und herabgesetzt ist. (Aus der Kinderklinik der Charitè Berlin).

\section{PRAKTISCHE ÜBER URÄMIE UND URÄMIEBEHANDLUNG. \\ Von \\ Dr. Max Rosenberg, Oberarzt. \\ Aus der I. Inneren Abteilung des städtischen Krankenhauses Chariottenburg-Westend (Direktor: Prof. F. UMBER).}

Der unter dem Namen Urämie in der Klinik zusammengefaßte Symptomenkomplex, seine Zugehörigkeit zu gewissen Formen der Nierenerkrankung, seine Abgrenzung von klinisch ähnlichen Krankheitsbildern, seine Einteilung und Klassifizierung, seine Ursachen und auslösenden Momente sind seit Traubes Zeiten Gegenstand einer sehr lebhaften Diskussion gewesen. Das Interesse an diesem Thema, wenn man es an der Zahl der sich mit ihm beschäftigenden Arbeiten messen darf, hat jetzt eher zu-als abgenommen, was nicht wundernehmen kann, wenn man bedenkt, daß das Studium der Nierenkrankheiten in den letzten Jahren an den verschiedensten Arbeitsstätten mit besonderem Eifer betrieben wurde, daß der Krieg das sonst zur Verfügung stehende Material vervielfältigte, und daß von den Erfahrungen, die aus diesem Studium hervorgingen, auch das Urämieproblem nicht unberührt blieb. Ich glaube es daher, trotz der Hochflut von Arbeiten, die sich in der letzten Zeit mit dem gleichen oder ähnlichen Themata befassen, wagen zu dürfen, einer Aufforderung der Schriftleitung dieser Wochenschrift nachzukommen und die prakŁisch wichtigen Ergebnisse für die Behandlung der Urämie, die sich aus der theoretischen Bearbeitung des Problems im letzten Jahrzehnt entwickelt haben, hier kurz zusammen. zufassen.

Um nicht mißverstanden zu werden, muß ich zunächst das, was ich im folgenden unter Urämie verstanden haben will, schärfer umgrenzen. Wie man auch über die Einteilung und Pathogenese đer Urämie denken mag, gerade für die Therapie hat sich eine, hauptsächlich von VoLHARD empfohlene Zweiteilung derselben fruchtbar erwiesen, wie es auch meines Erachtens unmöglich ist, diese 2 verschiedenen Formen, trotz gelegentlich zu beobachtender Vermischungen beider, klinisch urd ätiologisch unter einen Hut zu bringen. Es gibt eine Form der Urämie, bei der KrampfanfäIle verbunden mit längerem oder kürzerem Bewußtseinsverlust, verbunden mit plötzlich einsetzenden Sehstörungen, mit positivem Babinskischem Zeichen, eingeleitet von heftigsten Kopfschmerzen und Erbrechen und gefolgt von verschieden lange dauerndem Coma das charakteristische Symptom bilden. Diese Form der Urämie findet sich am häufigsten bei jugendlichen Individuen und bei akuten Nephritiden, und zwar bei solchen, die neben einer Blutdrucksteigerung erhebliche Odeme aufweisen oder gerade ausschwemmen. Das ganze Krankheitsbild gleicht, wie der ihm klinisch ähnliche epileptische Anfall, einer plötzlichen Entladung, einem plötzlichen Spannungsausgleich von langsam entwickelten Potentialdifferenzen. Häufig folgt dieser Urämieform, trotz dieser anscheinend schwersten Krankheitserscheinungen, eine schnelle Besserung aller nephritischen Symptome. Es ist, als ob sich bei zunehmender Schwüle und Windstille der Himmel mehr und mehr verfinstert, bis dann plötzlich ein Sturm und gleich darauf ein heftiges Gewitter mit Regen, Donner und Blitz losbricht, das in wenigen Minuten allen angesammelten Dunst und Hitze hinwegfegt und spült, so daß trotz des eben erst schwersten Wütens der Elemente der Himmel gleich darauf besonders klar und blau, die Luft besonders durchsichtig und rein und die Frische besonders angenehm und erquickend erscheint.

Gänzlich verschieden von dieser - sit venia verbo „Gewitterurämie" ist das Krankheitsbild derjenigen Urämie-

\section{ERGEBNISSE.}

form, die auch als echte Urämie, als Urämie im engeren Sinne, als Urämie im eigentlichen Wortsinne, wie wir sehen werden, bezeichnet wird. Es kommt hier, außer kurz vor dem Tode, zu keinem vollkommenen Bewußtseinsverlust, die Herabsetzung des Bewußtseins wird wohl am charakteristischsten mit dem Wort „Dösigkeit" ausgedrückt, es kommt hier zu keinen plötzlichen reinigenden Entladungen, es ist vielmehr das Bild einer schleichenden Vergiftung, das uns die Beobachtung des Krankheitszustandes aufdrängt, die Dösigkeit setzt allmählich ein, nimmt langsam zu, oder, wenn die Urämie abheilt, langsam ab. Es ist dies das Bild der Urämie, wie es besonders charakteristisch als Endstadium der Schrumpfnierenkrankheit beobachtet wird, aber auch bei akuten, häufiger subakuten Nephritiden, wenn auch oft in weniger charakteristischer Form vorkommt.

Die Diagnose dieser Urämie wird nach meinen Erfahrungen häufig zu Unrecht gestellt. Die Trias: Albuminurie, Blutdrucksteigerung und zerebrale Störungen müssen selbstverständlich den Verdacht einer Urämie wachrufen, aber sie rechtfertigen an sich noch nicht die Diagnose. Fehlt gar noch die Blutdrucksteigerung, und findet sich nur Benommenheit und Albuminurie bzw. Cylindrurie, wie es beispielsweise bei vielen exogenen Vergiftungen vorkommt, so ist die Annahme einer Urämie besonders unwahtscheinlich, da das Ausbleiben der Hypertonie sehr selten bei der Urämie beobachtet wird, und dann meist Folge einer erheblichen Kreislaufschwäche zu sein pflegt. Aber auch beim Vorhandensein einer Blutdrucksteigerung ist Vorsicht mit der Diagnose Urämie geboten. Vor allem eine Erkrankung wird am häufigsten fälschlicherweise für Urämie gehalten, und auf diese will ich deshalb kurz eingehen. Die vom 5. Lebensdezennium ab in der GroBstadt so häufige diffuse Arteriolosklerose (benigne Sklerose, Arteriolosklerosis incipiens, genuine Hypertonie), kann nämlich zu einem Krankheitsbilde führen, das die 3 genannten Symptome mit der echten Urämie gemein hat. Die Hypertonie, infolge der Arteriolosklerose aller Organe, die Albuminurie infolge der GefäBschädigung der Nieren, die zerebralen Erscheinungen infolge der GefäBerkrankung des Gehirns. Die letzteren können die für Urämie typische Dösigkeit täuschend nachahmen, so daß eine einmalige klinische Untersuchung die Diagnose echte Urämie nicht mit Sicherheit stellen oder ablehnen läßt, und eine Rest-N-Untersuchung des Blutes den differentialdiagnostischen Ausschlag geben muß. Im allgemeinen aber habe ich doch die Diagnose Urämie in solchen Fällen stellen sehen, bei denen die Art der zerebralen Ausfall- oder Reizerscheinungen gegen diese Erkrankung sprach. Es ist häufig nicht die charakteristische Dösigkeit, die solche Kranken aufweisen, es sind Verwirrungs- und Erregungszustände, Sprachstörungen, partielle Lähmungen, vollkommener Bewußtseinsverlust, usw., die nicht Folge einer schleichenaen Vergiftung sind, sondern durch ungenügende Blutversorgung des ganzen Gehirns oder einzelner Hirnteile, durch Erweichungsherde oder kleine Blutungen infolge der arteriosklerotischen Hirngefäßveränderung hervorgerufen werden. Aber selbst bei denjenigen seltenen Fällen, die das psychische Verhalten echter Urämiker kopieren, besitzen wir noch eine Anzahl rein klinischer differentialdiagnostischer Unterscheidungsmerkmale, die ich noch kurz hervorheben möchte.

Zunächst sei auf den in der Praxis nicht voll gewürdigten eigentümlichen urinähnlichen Geruch (UMBER) hingewiesen, den fast alle echten Urämiker aufweisen. Etwas Vorsicht ist allerdings auch hier insofern am Platze, als bisweilen Kranke mit sehr ungepflegtem Munde, einen ähnlichen, bisweilen nicht sicher unterscheidbaren Geruch der Ausatmungsluft aufweisen 